\title{
Study of Effects of Interfaces in the Propagation of the Energy by Optical Modes in Coaxial Cylinders
}

\author{
Eloneid Felipe Nobre*, Ricardo Sávio Teixeira Moretzsonh*, \\ Rivelino Cunha Vilela ${ }^{\dagger}$, G.A. Farias*, and N.S. Almeida* \\ *Departamento de Física, Universidade Federal do Ceará, Caixa Postal 6030, 60455-760 Fortaleza - Ce, Brazil \\ ${ }^{\dagger}$ Centro Federal de Educação Tecnológica (CEFET), Av. Newton Bello, Vila Maria, 65919-050, Imperatriz-Ma, Brasil
}

\section{Received on 8 December, 2005}

\begin{abstract}
We study the influence of the interfaces on the dispersion relation, energy and power flow of polaritons propagating in coaxial cylinders. We consider an infinite coaxial cylinder of internal and external radii designated by $a$ and $b$, respectively, submitted to a magnetic dc field applied parallel to the z-axes. The presence of a magnetic field causes significant alterations in the modes of propagation of polaritons. The numerical results are obtained for the surface polaritons propagating in semiconductors cylinders of GaAs in presence of magnetic fields of 5 kG.
\end{abstract}

Keywords: Cylindrical interface; Polaritons in Cylindrical structures; Localized polaritons

\section{INTRODUCTION}

The properties of polaritons has been studied since many years by many authors in different geometries [1-3]. However, the recent development of new techniques of producing micron-size materials, the intense current interest in lefthanded materials, and the enhanced transmission through subwavelength holes updated the interest on the study of this kind of electromagnetic excitation. Particularly, the study of polaritons in microcavities has received a great deal of attention nowadays. Surface plasmon polaritons play a particularly important role in light-matter interactions at the nanometer scale and they are important for applications in biology, nanotechnology, and materials sciences [4 - 9].

The change of the geometry, from plane or spherical to cylindrical, induces considerable effects on the properties of the polaritons. Some of these changes were object of study in the last decade [10] but the complete comprehension of the behavior of these modes in this geometry is far away to be reached. Recently, due to the possibility of to grow micronsize materials with shapes to fit different technological needs, the interest of the study of electromagnetic excitation in materials with cylindrical geometry was renewed [11].

Materials with this symmetry are important for the correct use of the scanning near-field optical microscopy (SNOM) which rely on the use of nanometer-sized probes. These probes are generally sharpened optical fibers that have a cylindrical geometry. It should be remarked that a good understanding of the physical behavior of these modes is of fundamental importance for gaining a proper interpretation of the experimental data obtained by these optical devices. For instant, it was found that evanescent light field plays an important role in the interaction with neutral atoms confined on glass surface surrounding a hollow fiber. Therefore, the results obtained by, for example, scanning near-field microscopy (SNOM) would be better understood if the physical behavior of these modes is completely understood for the different SNOM configurations.

This work studies the influence of interfaces on the dispersion relation, propagation of energy and power flow of polari- tons propagating in coaxial hollow cylinders. As can be seen bellow, there are significant changes if the system under consideration is a massive or coaxial cylinder. Similarly to the planar geometry, in the coaxial cylinder, the presence of interfaces causes the appearance of new branches in the dispersion relation and introduce alterations in the physical behavior of the energy, power flow and the velocity of transport of energy, in comparison with those obtained for a massive cylinder [12].

\section{THEORY}

The system studied is an infinite coaxial hollow cylinder of internal and external radii designated by $\mathbf{a}$ and $\mathbf{b}$, respectively. The geometry of the system defines three different regions: Region I $(0<r<a)$, region II $(a<r<b)$ and region III $(r>b)$. For the applications in this work, we considered the region II as the only optically active region. Regions I and III, are considered homogeneous, isotropic and in the numerical applications were considered as the vacuum. The system is submitted to an external dc magnetic field applied parallel to the $\mathrm{z}$-axis of the cylinder. The presence of a magnetic field applied on a semiconductor cylindrical system, causes significant alterations in the modes of propagation of polaritons. One of the main effects of the application of the field is to promote the decoupling between the electric transverse (TE mode; $\left.\mathrm{E}_{z}=0\right)$ and the magnetic transverse (TM mode; $\mathrm{H}_{z}=$ $0)$ modes, by the elimination of the TE mode [2].

Solving the Maxwell's equations in cylindrical coordinates and considering the continuity of the tangential components of the electromagnetic fields at the interfaces of the cylinder, we obtain the dispersion relation which gives us information on the modes in order to obtain the energy flow, the energy density and the total power.

The external magnetic field provokes remarkable changes in the dielectric function of the material, which is usually represented by a diagonal matrix. It introduces off-diagonal terms in the dielectric tensor and, consequently, causes dramatic changes in the physical behavior of the polaritons. The non-diagonal dielectric tensor is now given by: 


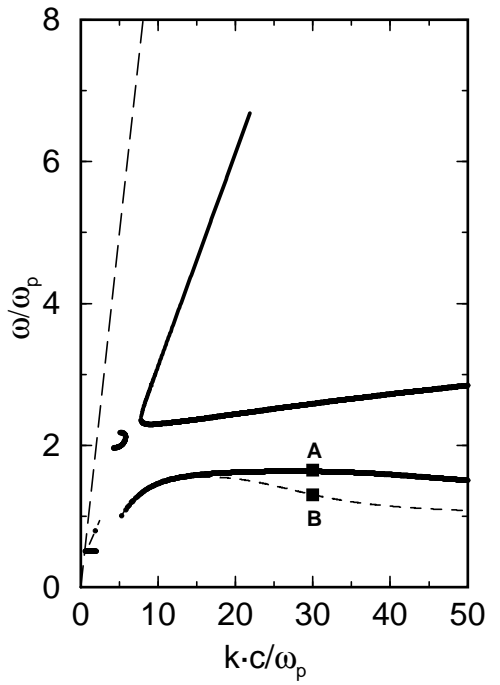

FIG. 1: Dispersion relation for surface polaritons propagating in two different hollow cylinders, whose external radius, $b=50 \mu \mathrm{m}$, is kept unmodified. The solid line represents the modes for the cylinder whose internal radius is $\mathrm{a}=10 \mu \mathrm{m}$. The dashed lines represents the case of $\mathrm{a}=25 \mu \mathrm{m}$. The long dashed line is the light line.

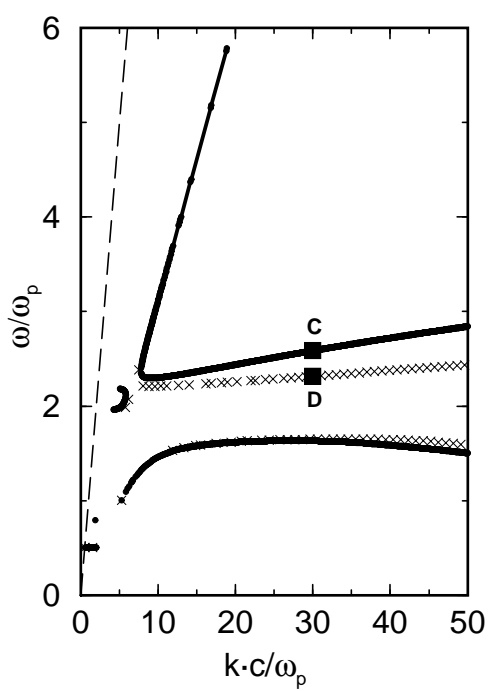

FIG. 2: Dispersion relation for surface polaritons propagating in two different hollow cylinders, whose internal radius, $\mathrm{a}=10 \mu \mathrm{m}$, is kept unmodified. The solid line represents the modes for the cylinder whose external radius is $\mathrm{b}=50 \mu \mathrm{m}$. The $\mathrm{x}$-lines represents the case of $\mathrm{b}=20 \mu \mathrm{m}$. The long dashed line is the light line.

$$
\overleftrightarrow{\varepsilon}(\omega)=\left(\begin{array}{lll}
\varepsilon_{1 j} & -\varepsilon_{2 j} & 0 \\
\varepsilon_{2 j} & \varepsilon_{1 j} & 0 \\
0 & 0 & \varepsilon_{3}
\end{array}\right)
$$

The index $\mathrm{j}$ refers to region (II is the optically active medium). The frequency dependent elements of the dielectric tensor at the region II are given by:

$$
\begin{gathered}
\varepsilon_{1 I I}=\varepsilon_{\infty}\left\{1+\frac{\omega_{p}^{2}}{\omega_{c}^{2}-\omega^{2}}\right\}, \\
\varepsilon_{2 I I}=i \varepsilon_{\infty}\left\{\frac{\omega_{c} \omega_{p}^{2}}{\omega\left(\omega_{c}^{2}-\omega^{2}\right)}\right\}, \\
\varepsilon_{3 I I}=\varepsilon_{\infty}\left\{1-\frac{\omega_{p}^{2}}{\omega^{2}}\right\},
\end{gathered}
$$

In the equations above, $\omega_{p}$ and $\omega_{c}$, are the plasma and cyclotron frequencies, respectively:

$$
\omega_{p}^{2}=\frac{4 \pi n e^{2}}{m_{e}^{*} \varepsilon_{\infty}}, \omega_{c}=\frac{e B_{0}}{m_{e}^{*}}
$$

where $\mathrm{n}$ and $m_{e}^{*}$ are the concentration and the effective mass of the carriers, $\mathrm{e}$ is the magnitude of electron charge, and $\mathrm{B}_{0}$ is the intensity of the externally applied magnetic field.

The components of the electromagnetic field are obtained in terms of the modified Bessel Functions and we can show that for TE modes $\left(E_{z}=0\right)$ only the trivial solution $\mathbf{E}=\mathbf{0}$ and $\mathbf{H}=\mathbf{0}$ can be found. Consequently, only the TM modes $\left(\mathrm{H}_{z}=0\right)$ can propagate.

The energy flow is calculated by the time average of the Poynting vector, whose integral in all regions gives us the total power flow. The energy density is obtained considering the case of an anisotropic medium.

\section{RESULTS AND DISCUSSIONS}

The results presented bellow were obtained for hollow cylinders which has GaAs as the optically active material (region II) in presence of an externally applied magnetic field of $5 \mathrm{kG}$.

In the Fig. 1, we show the dispersion relation for surface polaritons propagating in two different hollow cylinders with the same external radius $\mathbf{b}=50 \mu \mathrm{m}$ and the internal radius $\mathbf{a}$ equal to of $10 \mu \mathrm{m}$ and $25 \mu \mathrm{m}$. In Fig. 2, we keep unmodified the internal radius $\mathbf{a}=10 \mu \mathrm{m}$ and the external radius $\mathbf{b}$ assumes the values of $20 \mu \mathrm{m}$ and $50 \mu \mathrm{m}$. In both figures we consider the magnetic field $\mathrm{B}_{0}=5 \mathrm{kG}$. The mathematical functions, from which we obtain the dispersion relation, are obtained from the usual boundary conditions for the electromagnetic fields at the interface of the regions; therefore they are functions of the radius of the cylinder layers. So when we analyze the dispersion relation curves, in general there is no condition to predict "a priori" the region where the mode is propagating. However, in the Figs. 1 and 2 we can observe clearly the effects of the interfaces. In the Fig. 1, where it is changed the internal radius a while the external radius b remains unmodified, the lower branch shows alterations while the higher one do not exhibit noticeable changes. Moreover, is observed that the modes are 
moved to the low frequencies region when the internal radius is increased. The opposite behavior is observed in the Fig. 2, where it is modified the external radius $\mathbf{b}$ while the internal radius a is kept constant. The higher branch is moved to the high frequencies region while the lower branch remains almost unmodified when the the external radius of the cylinder increases.

The energy flow is shown in Figs. 3 and 4 for the modes assigned in the dispersion relation curves of the Figs. 1 and 2. The maximum of intensity of the energy flow occurs in the internal region II. Depending on the frequency of the polariton (dispersion relation branch), we can observe a maximum of this quantity near of the internal or of the external interfaces of the region II. It seems that modes that propagate with high frequencies "prefer" the external interface and on other way around, those of lower frequencies propagate near the internal interface.

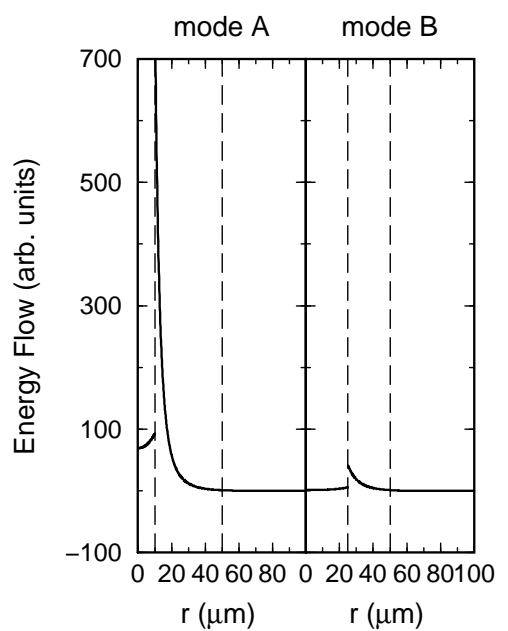

FIG. 3: Energy flow for modes indicated by letters $\mathbf{A}$ and in Fig. 1. The dashed lines indicate the interfaces of the cylinders.
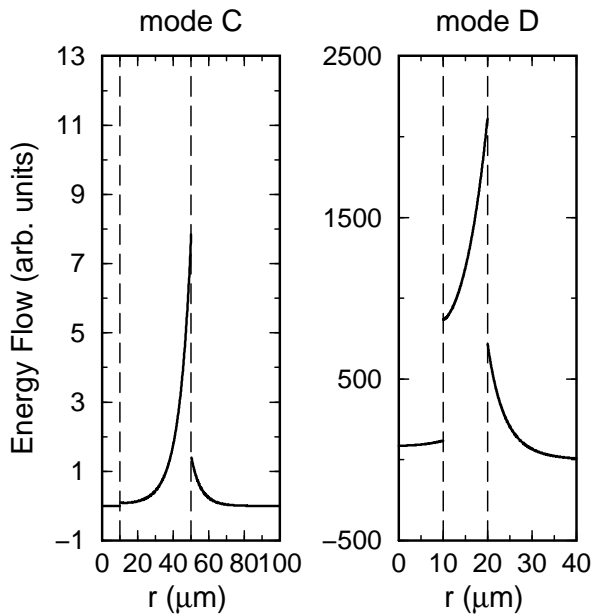

FIG. 4: Energy flow for modes indicated by letters $\mathbf{C}$ and $\mathbf{D}$ in Fig. 2. The dashed lines indicate the interfaces of the cylinders.
The energy density is showed in Fig. 5 for the mode B pointed out in Fig. 1. We can see that it presents a behavior similar to the energy flow. In regions I and III, the energy density is almost equal to zero and it is concentrated inside of the optically active part of the cylinder.

In Fig. 6 we show the total power propagated by surface polaritons in cylinder of internal and external radii equal to 25 $\mu \mathrm{m}$ and $50 \mu \mathrm{m}$, respectively. It is obtained by integration of energy flow in all regions. We notice that it exhibits a maximum value for modes with small wavevectors for all cylinders analyzed.

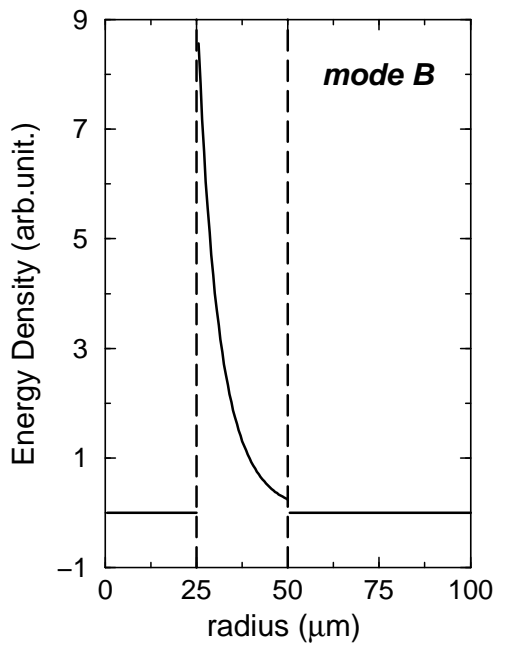

FIG. 5: Energy density for mode B, indicated in Fig. 1. The cylinder considered has internal radius $\mathrm{a}=25 \mu \mathrm{m}$ and external radius $\mathrm{b}=50 \mu \mathrm{m}$. The dashed lines indicate the interfaces of the cylinder.

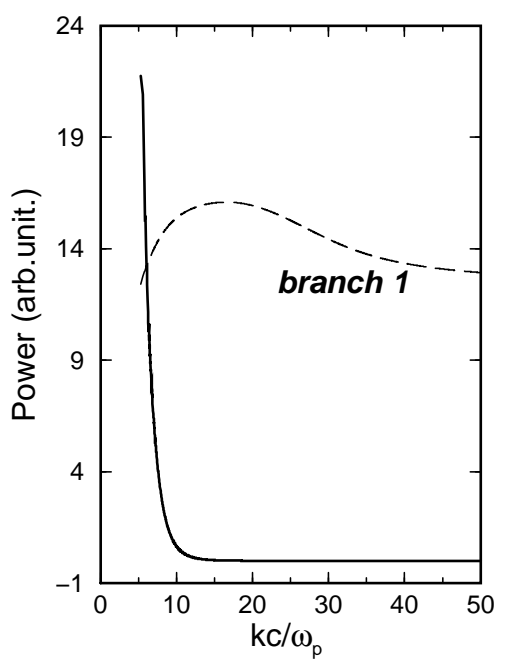

FIG. 6: Total Power for surface polaritons propagating in cylinder of internal radius $a=25 \mu \mathrm{m}$ and external radius $\mathrm{b}=50 \mu \mathrm{m}$. The dashed curve represents the branch of the dispersion relation whose modes we have analyzed. 


\section{FINAL COMMENTS}

It is already well know the rich variety of the physical characteristics of polaritons propagating in materials with cylindrical geometry. In this work we show that, in a way similar to the multilayer planar systems, the presence of interface introduce new features which are the result of the combined effect of the geometry and the optical characteristic of the constituent material. As can be seen in the Fig. 3, for example, the appropriate choice of the internal radio is the main responsible to localize the energy flow at a particular region. We hope these results can motivate experimental verification of our theoretical predictions.

\section{Acknowledgement}

G. A. Farias and N. S. Almeida are partially supported by CNPq and Fundação Cearense de Pesquisa e Cultura (FUNCAP).
[1] C. A. Pffeifer, E. N. Economou, and K. L. Ngai, Phys. Rev. B 10, 3038 (1974).

[2] H. Khosravi, D. R. Tilley, and R. Loudon J. Opt. Soc. Am A 8 112 (1991).

[3] G. A. Farias, E. F. Nobre, R. Moretzsohn, N. S. Almeida, and M. Cottam, J. Opt. Soc. Am. 19 2449, (2002).

[4] Pendry, J. Nature, 423, 22-23.( 2003)

[5] (a) William L. Barnes, Alain Dereux \& Thomas W. Ebbesen, Nature, 424, 824, (2003). (b) Altewischer, E.; van Exter, M. P.; Woerdman, J. P. Nature 418, 304-306 (2002). (c) Ditlbacher, H.; Krenn, J. R.; Schider, G.; Leitner, A.; Aussenegg, F. R. Appl. Phys. Lett. 81, 1762-1764. (2002),

[6] Rothenhausler, B.; Knoll, W. Nature 332, 615-617.(1988)

[7] Baksh, M. M.; Jaros, M.; Groves, J. T. Nature 427, 139 (2004)

[8] Krenn, J. R.; Weeber, J. C. Philos. Trans. Royal Soc. London, Ser. A 362, 739 (2004).

[9] Hecht, B.; Bielefeldt, H.; Novotny, L.; Inouye, Y.; Pohl, D. W.
Phys. ReV. Lett. 77, 1889 (1996)

[10] (a) M. A. Kaliteevski, S. Brand, R. A. Abram, V. V. Nikolaev, M. V. Maximov, N. N. Ledentsov, C. M. Sotomayor Torres, and A. V. Kavolin, Phys. Rev. B 61, 13791 (2000). (b) M. A. Kaliteevski, R. A. Abram, and V. V. Nikolaev, J. Mod. Opt 677 (2000). (c) D. Liballoy, H. Benisty, C. Weisbush, T. F. Kraus, C. J. Smith, R. Houdre, and U. Oesterle Appl. Phys. Lett. 73, 1314 (1998)

[11] (a) H. Khosravi, D. R. Tilley, and R. Loudon, Opt. Soc. A 8, 112 (1991). (b) G. C. Aers, A. D. Boardman, and B. V. Paranjape, J. Phys. F, 10, 53 (1980). (c) H. Ito, T. Nakata, and M. Ohtsu, K. I. Lee and W. Jhe, Phys. Rev. Lett. 76, 4500 (1996).

[12] (a) E. F. Nobre, R. N. Costa Filho, G. A. Farias, and N. S. Almeida, Phys. Rev. B 57, 12275 (1998). (b) E. F. Nobre, G. A. Farias, and N. S. Almeida, J. Opt. Soc. Am. A 17, 173 (2000). 